\title{
Study on Relay Protection of Process Layer and Substation in Relay Protection of Intelligent
}

\section{Substation}

\author{
KONG Hai-bo, XIE Xiao-dong, WANG Ling-fei, SUN Peng, XU Bing
}

\begin{abstract}
The relay protection facilities of the intelligent substation are the basic elements of modern power system construction. Based on the principles of flexibility, safety and accuracy, it can be divided into process layer and substation layer according to specific equipment. The former is suitable for single sampling of disposable equipment, which can be separated from the base station synchronization sampling, with synchronous digital system and ping-pong algorithm to achieve the overall sampling of synchronization, that is, the distribution configuration to protect the various components, through a separate transmission medium to achieve information transmission; For the substation components centralized backup protection, take the online real-time tuning technology.
\end{abstract}

Key words: Power system; intelligent substation; relay protection

\section{Introduction}

Compared with the general substation, the use of intelligent substation is growing, and the technology of relay protection is endless, but so far the exploration of system structure is relatively few. Whether it is intelligent substation or general substation, the system structure is similar, it turns out that this system structure can effectively protect the substation work safety and reliability, but also to protect the current power equipment, the overall coherence. In general, compared with the general substation, the new intelligent substation uses fiber optic cable as the transmission carrier, and the optical signal based on the coding technology replaces the previous electrical signal, which guarantees the real-time validity of the sampling and the timeliness of the information feedback.

In addition, the protection configuration continues the advantages of general relay protection, for all power components set up a rapid trip, each stage of the auxiliary backup protection. However, now on this part of the protection settings, not completely combined with the specific characteristics of the intelligent substation to properly adjust and improve.

Accordingly, this paper designed a set of intelligent substation relay protection configuration plan, substation equipment is divided into process layer and substation layer. The former is suitable for single sampling of disposable equipment, can be separated from the base station synchronization sampling to ensure the distribution of installation and dual equipment; the latter for the power components of the centralized backup protection, unified set of power system voltage based on self-sensing and timely maintenance technology and has a wide area protection interface.

\section{The configuration of relay protection for in- telligent substation}

On the intelligent substation relay protection configuration, the main object includes the process layer and the substation layer. The former is mainly responsible for a separate protection of the equip- 


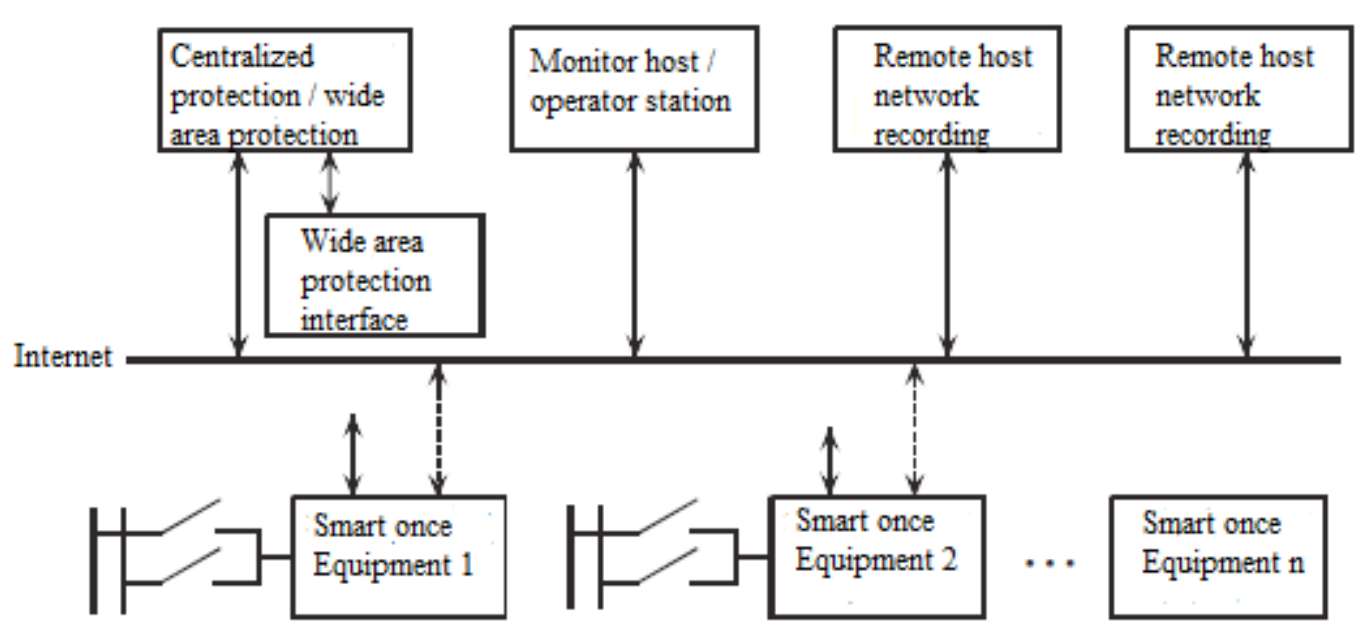

Figure 1 Intelligent substation relay protection configuration diagram

ment, divided into two types: first, a device is a smart device, the equipment installation from the protective effect; second, if the old equipment to improve, should be repaired, Control cabinet, so as to ensure the normal operation of equipment and follow-up maintenance. GOOSE and sampling values are sent via Ethernet to receive, based on IEEE1588 guarantee timeliness. Intelligent substation relay protection configuration is shown in Figure 1 below.

According to the principle of distributed installation of the base station, it can simplify the protection of the relay station and ensure the minimum distance between the substation equipment and the secondary equipment, and avoid the protection failure caused by trip- ping and sampling. In addition, the entire substation data acquisition and processing rely on Internet monitoring, testing and other functional consumption, and relay protection to reduce this part of the consumption.

\section{On the relay protection of the process layer}

In general, the process layer protection for sudden tripping of the line protection, such as circuit longitudinal, bus differential, substation layer backup protection through the centralized device to achieve, is mainly responsible for the main protection. Based on this, the backup protection device can be simplified to achieve the goal of system hardware simplification. In addition, the protection of the value of the equipment set more stable, basically does not change with the working

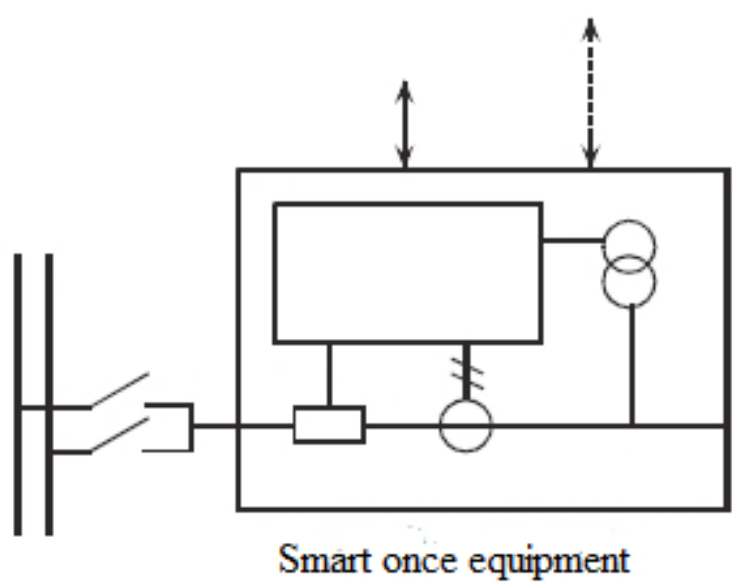

Figure 2 Schematic diagram of single-circuit circuit protection 


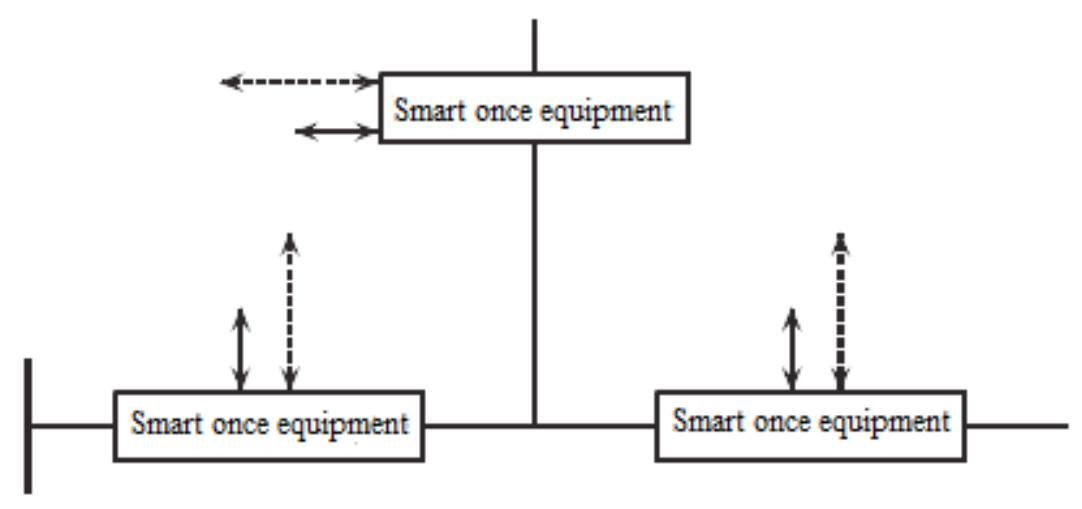

Figure $33 / 2$ line connection form line protection diagram

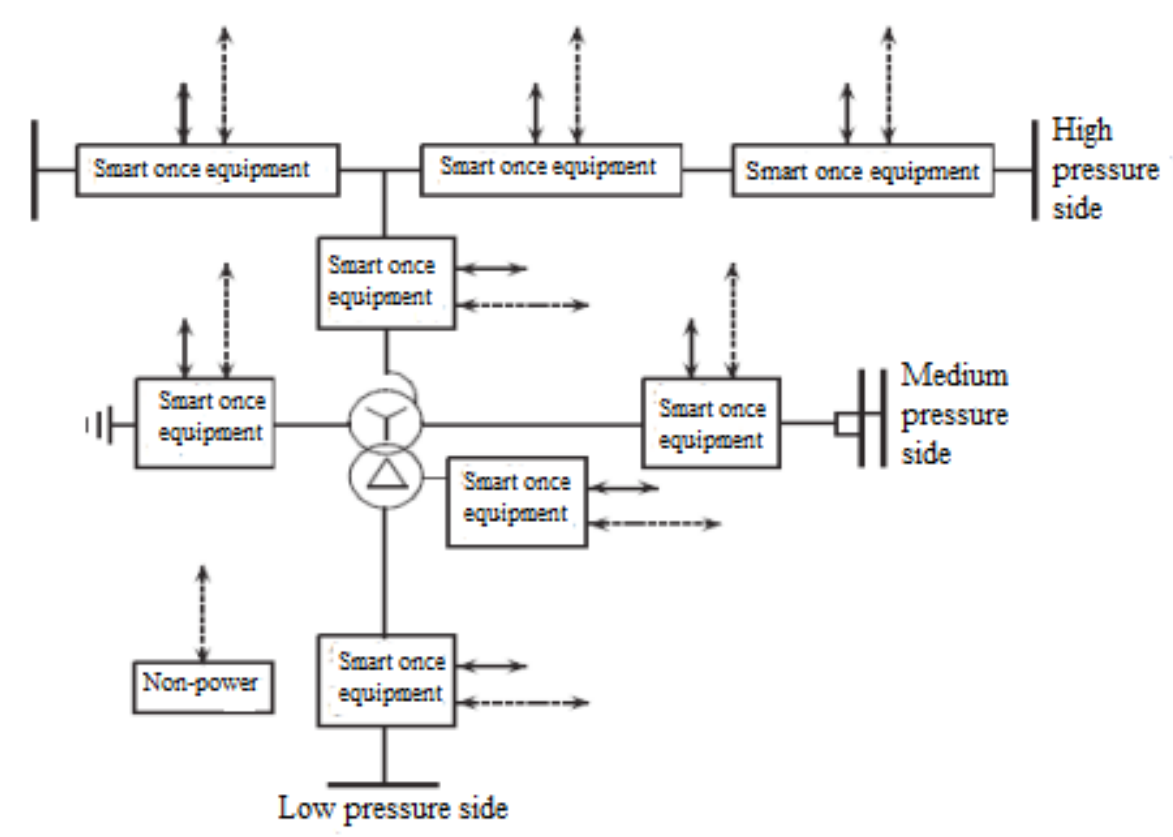

Figure 4 2/3 line connection of the transformer equipment protection diagram

conditions of the power system changes.

However, because of the principle of independence of relay protection, after the process layer and substation layer protection equipment is centralized, if only one switch to protect the circuit and bus, then the equipment should be independent, can be set as a separate functional components. The following is a detailed analysis of the process layer protection function.

\subsection{Circuit protection function}

With regard to the circuit protection function in the process layer, the main protection is designed according to the principle of the longitudinal path or the longitudinal differential, and the backup protection device is integrated into the protection device. For single-circuit main wiring, circuit protection is the use of optical fiber communication main protection and line protection to achieve, that is, vertical protection, see Figure 2 below.

In the case of a $3 / 2$ line connection, the value of the switching current and the switching current on the series line should be collected, as shown in Figure 3 below. According to the principle of circuit collocation, integrated protection equipment without collecting voltage, but for some special work or protection program need to collect voltage value. In this case, the voltage value can be collected separately, and it is connected with the main protection data transmission line to complete its acquisition with the current value. Because with the same circuit, so the switch current value can be collected separately, and then through 


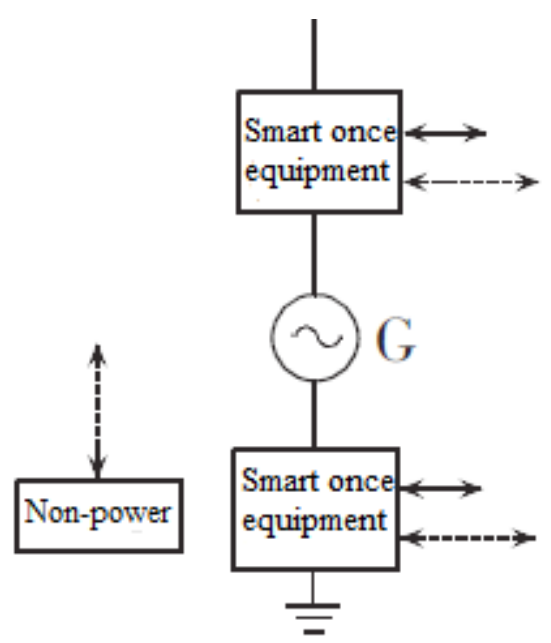

Figure 5 Schematic diagram of reactor protection

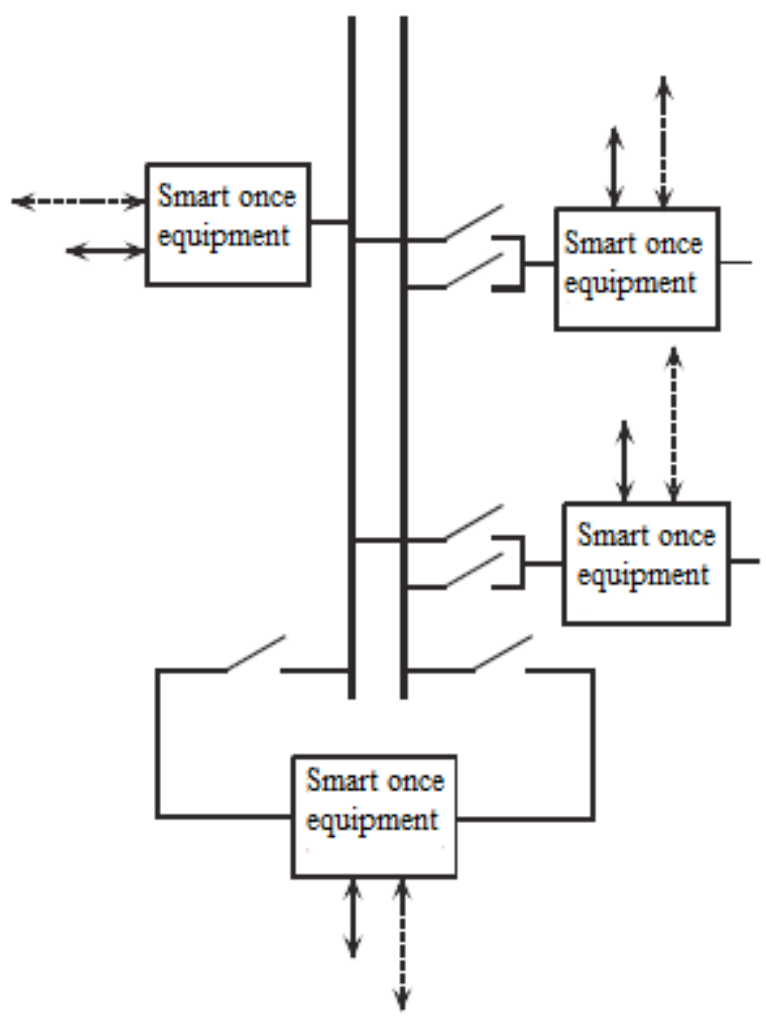

Figure 6 Schematic diagram of bus protection

the main protection data transmission line to achieve data synchronization, and the way the main protection of data transmission line standards higher.

\subsection{Transformer equipment protection function}

For the protection of transformer equipment, should be based on the principle of differential protection, to take the protection of the distribution device, the same, the backup protection is still taken integrated device.
For non-power protection, it should be installed independently, with a cable connected to the circuit breaker trip, and the trip command is connected to the GOOSE computer with a cable connection. 2/3 line connection of the transformer equipment protection diagram shown in Figure 4.

2.3 Reactor protection function 


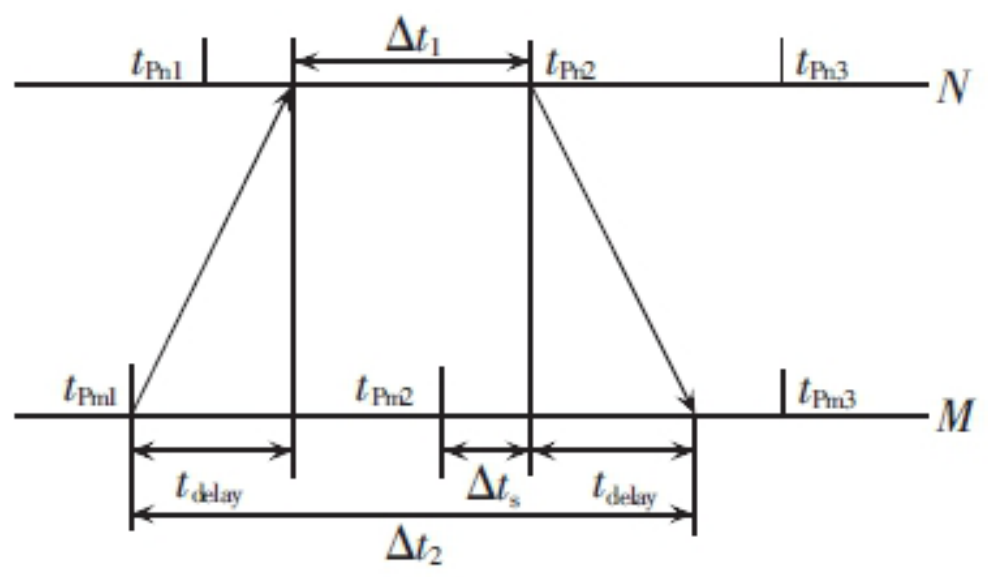

Figure 7 Sampling numerical modification technology application diagram

Generally speaking, the reactor protection and transformer equipment protection is roughly the same. The protection of the reactor is shown in Figure 5 below.

\subsection{Bus protection function}

On the relay bus protection, but also the distribution of the installation of protective devices, each part of the realization of separate bus protection purposes, in the event of failure, only the fault part of the circuit trip. In addition, functional failure protection is taken in an integrated form of protection. The busbar protection diagram is shown in Figure 6 below.

2.5 To take synchronization protection
For the substation function protection device, should be regarded as multi-terminal line protection, and through the synchronization protection to achieve the synchronization of the sample value acquisition. And based on the simplicity of the principle of installation, should be an effective use of ping-pong principle. This principle requires that the circuit head and end protection devices collect the sample values separately at the same frequency and keep the time to send and receive data. At present, China's general adoption of ping-pong principle-based synchronization technology is divided into sampling numerical modification technology and sampling time adjustment technology.

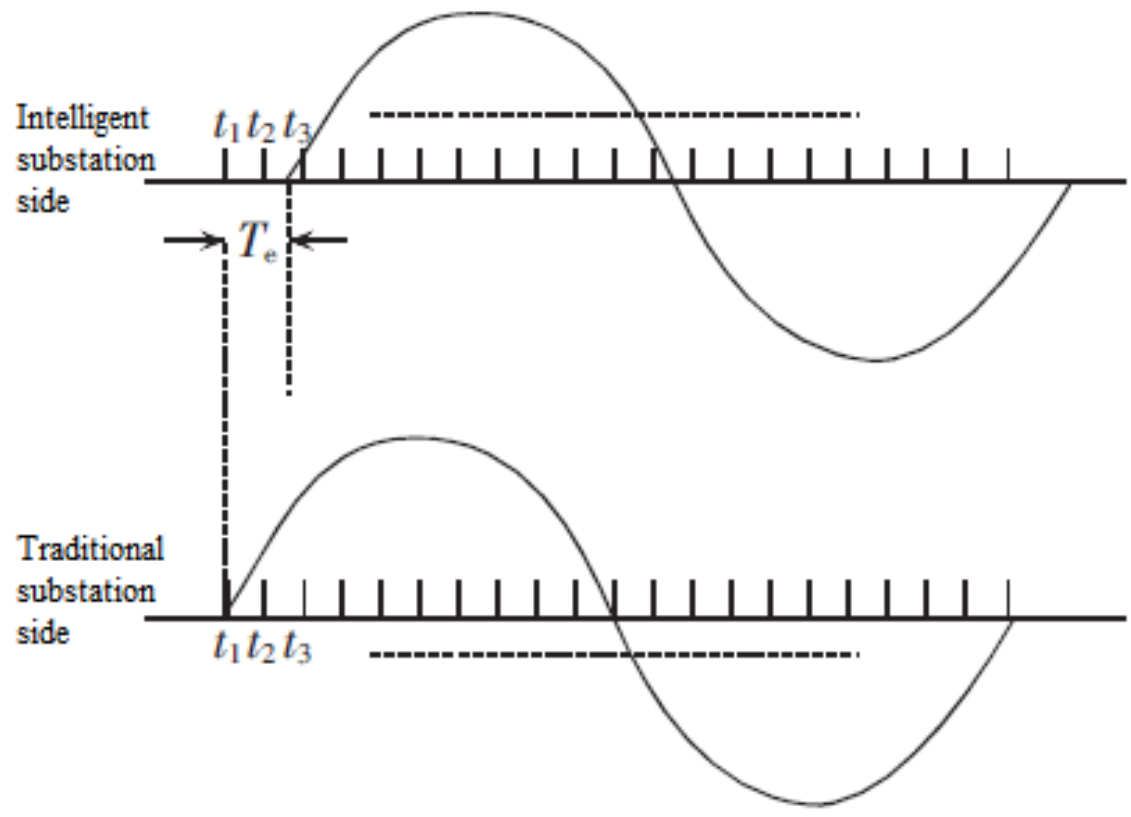

Figure 8 Delay adjustment diagram 
Sampling numerical modification technology, also known as synchronous vector technology, the basic synchronization principle shown in Figure 7 below. M and $\mathrm{N}$ are two opposite sides, the information transmission period is $T, t_{P m 1}, t_{P m 2}, t_{P n 1}, t_{P n 2}$ is the opposite side of the numerical sampling time, $\Delta \mathrm{t}_{1}, \Delta \mathrm{t}_{2}$ is the side of the opposite information and the side of the previous information transmission time difference, $T_{\text {delay }}$ is the length of time the information is transmitted between the two sides. The opposite side of the data sent before the $M_{1}$ and the front of a $\Delta t_{1}$, if the information on the two sides is equal to each other, the ta $=\left(\Delta \mathrm{t}_{2}+\right.$ $\left.\Delta \mathrm{t}_{1}\right) / 2$, ta is the same as the opposite Pn2 information, and the difference between the two sides is the same as the opposite Pn2 information. $\left.\left(t_{P m 2}-t_{P m 1}\right)\right]$, the relative angle is $\Delta \theta$, and the transmission line extension time amount $\mathrm{t}_{\text {delay }}=\left[\Delta \mathrm{t}_{2}-\Delta \mathrm{t}_{1}\right] / 2$. The time interval is $t_{P m 2}$, and the time interval difference $\Delta t_{s}=$ [ta- $\left(t_{P_{m} 2}\right.$ $-t_{\left.P_{1}\right)}$ ). The synchronization technology to ensure that the beginning of the line and the end of the sampling of a single line, while analyzing the transmission line to adjust the information transmission and reception delay $t_{\text {delay' }}$ it can be seen, if the transmission line by external interference, generally do not have a synergistic sampling effect.

In addition, the sampling time adjustment technology to ensure the independence of the substation sampling, combined with the station sampling time and timely control to ensure that both sides of the line synchronization sampling height and accurate.

Based on the principle of simplification of the protection device, the protection device for realizing the synchronization function is divided into:

$\square$ The information reception interruption in the optical fiber transmission line is independent of the information transmission interruption. In the previous protection system, these two parts are the same interrupt control, for the electronic sensing device, the information is sampled through the sampling part, and the information sent in the transmission section, caused by the delay, can be obtained by measurement.

Control the adjustment of both sides of the information transmission time. With the help of ping-pong synchronization principle of sampling time adjustment technology to ensure that both sides of information synchronization, to achieve both sides of the protection device information transmission time consistency.

For the sampling delay and transmission delay adjustment, can be used to analyze the following Figure 8 to ensure that both sides of the time to receive infor-

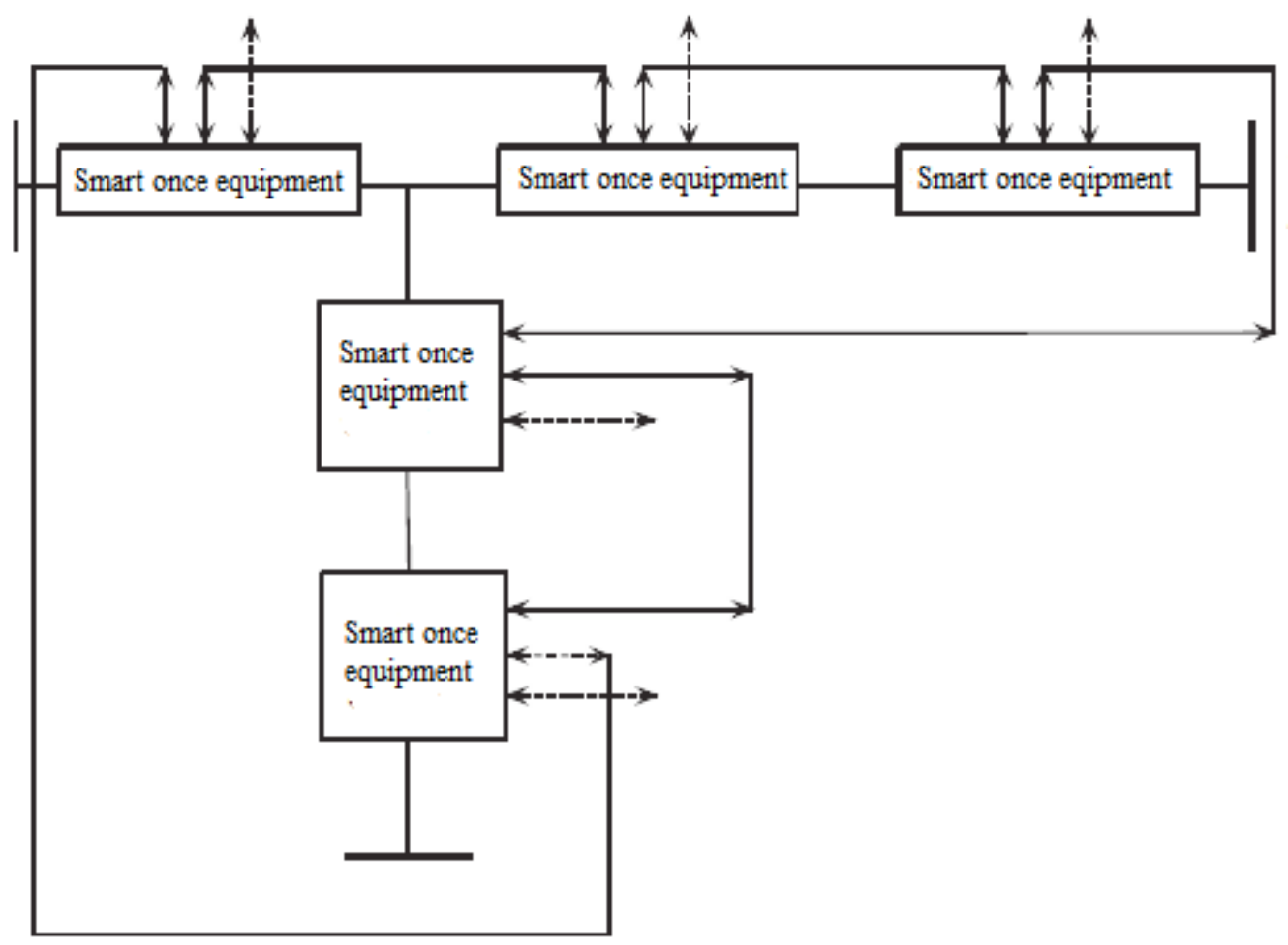

Figure 9 Synchronous data transfer cycle diagram 
mation synchronization.

\subsection{Distributed protection of data transmission}

At present, the distributed protection data transmission takes the SDH system, and the data transmission protection is set to the transmission cycle. Side through the $3 / 2$ line connection, the other side is a single circuit connection to achieve the purpose of protection, see Figure 9 below.

According to the diagram shown in the transmission cycle diagram, there are two kinds of design:

First, the data transmission synchronization in the substation is only used for the synchronization function, its network information transmission speed is not high. The collected sample values are spread over Ethernet, so the power equipment can access the required information online. In practice, this mode is only used for synchronous redundancy design.

Second, the entire relay protection device not only to ensure data synchronization, but also to achieve the data to send and receive, in considering the bus at the maximum time difference, based on the need to achieve within 1-millisecond interval information transmission. In addition to the program with a redundant design, but also set the information transmission function.

Synchronization system can be a protection device as the main protection, and auxiliary protection to achieve its transmission interrupt synchronization.

\section{On relay protection of substation layer}

The substation layer is mainly designed for the centralized protection of the backup system, and the voltage of each part of the power system is unified, and the protection configuration is realized based on the self-sensing and timely maintenance technology.

Generally speaking, the system in addition to the main transformer substation for the back of the backup and switch protection, but also on the auxiliary substation remote backup protection. It can be seen that the protection of any substation content includes: $\square$ Near backup protection mainly for the substation of the bus and other lines; $\square$ Far backup protection of the object is the connection side of the bus and other terminals connected.

In fact, the backup protection system is mainly responsible for the collection and analysis of the main substation line basic data, the basic situation of the circuit breaker equipment, while receiving the surrounding auxiliary substation short circuit, the basic line data. Combined with the detected information data, in a timely manner to determine the power system error components, and feedback to the effective circumvention program. In addition, through the offline value setting calculation method, in accordance with the circuit work path in advance to identify the corresponding algorithm plan, and substation protection configuration in accordance with the specific power system parameter values clear a variety of working paths, the protection configuration is also adjusted to set the value within the scope, and then achieve the goal of protection action performance. In addition, the system should also include a low voltage or low-frequency protection, overload shutdown lines and other configurations.

\section{Conclusion}

Compared with the general substation, the use of intelligent substation is growing, and the technology of relay protection is endless, but so far the exploration of system structure is relatively few. For the substation circuit, transformer equipment, bus and other components, the effective protection of the design, distributed in the form of installation to the intelligent device, the system protection settings to simplify and reduce the relay protection detection and maintenance difficult. In addition, for the backup protection, integrated configuration, to ensure mutual protection of substation protection.

\section{References}

[1] ZHANG Zhi-Rui, XIAO Fan, JIAO Shao-Lin, DONG Wen-Xiu, WANG Feng, ZHANG Zhe, YIN Xiang-Gen. Reliability Analysis of Protection System for Different Process Layer Network Structures [J]. Power System Protection and Control.

[2] Huang Minghui, Shao Xiangchao, Zhang Chi, Wang Haizhu, Li Yiquan, Cai Zexiang. Based on OPNET Intelligent Substation Relay Protection Modeling and Simulation [J]. Power Automation Equipment. 2013 (05)

[3] Wang Chao, Wang Huifang, Zhang Chi, Liu Wei, Li Yiquan, He Ben Teng. Study on Reliability Modeling of Relay Protection System in Digital Substation [J]. Protection and Control of Power System. 2013 (03)

[4] Liu Ning, Zhou Wu, Cai Ming, Li Jun, Chen Wuchen, Xu Guangwei.Study and application Of The Performance Evaluation of the Secondary Equipment of 
Intelligent Substation [J]. Automation and Instrumentation 2016 (03)

[5] Chen Xiajie. Intelligent Substation Protection Device Trip Mode Comparison [J]. Electric Power and Electricity. 2011 (04)

[6] Fan Chen, Ni Yimin, Dou Renhui, Shen Jian, Gao Chunlei, Huang Guofang.Analysis of Process Network of Intelligent Substation [J]. Automation of Electric Power Systems 2011 (18)

[7] HOU Wei-hong, ZHANG Pei-chao, HU Yan, SU Yong-chun. Protection System based on High Availability Automation Network and its Reliability Analysis [J]. Power System Protection and Control. 2010 (18)

[8] ZHU Lin, CHEN Jin-fu, DUAN Xian-zhong.Improvement of Reliability of Redundant Substation and its Reliability and Economic Evaluation [J]. Journal of Electrical Engineering 2009 (10)

[9] ZHANG Pei-chao, GAO Xiang.Analysis of Reliability and Component Importance of All-Digital Protection System [J]. Proceeding of the CSEE, 2008 (01)

[10] Huo Chao, Zhang Peichao. Electronic Protection System to Consider the Economic Importance of Com- ponent Analysis [J]. Power System Automation. 2007 (13)

[11] Liu Ning, Zhou Wu, Cai Ming, Li Jun, Chen Wu, Xu Guangwei. Research and Application of Operation Condition Evaluation of Secondary Equipment of Intelligent Substation [J]. Automation and Instrumentation 2016 (03)

[12] Du Shuangyu, Wang Xianpei, Xie Guangbin, Cai Tao, Zhang Qilin. Reliability Evaluation of Substation Automation System based on IEC61850 [J]. Protection and Control of Power System 2012 (05)

[13] ZHU Sheng-hui, ZHOU Fu-qiang, XIA Han, NI Zhen-qiang.Study on Relay Protection Means of Intelligent Substation Based on Spatial Dimension [J]. Automation and Instrumentation 2016 (02)

[14] Shen Yuan. A N-k Risk Method for Multi-mode Concealment Fault of Relay Protection System [J]. Automation and Instrumentation 2015 (11)

[15] ANDERSSON L, BRAND K P, BRUNNER C, et al.Reliability Investigations for SA Communication Architectures Based on IEC61850. Proceedings of IEEE Power Tech 2005. 2005 